\title{
Suppressed expression of LDHB promotes age- related hearing loss via aerobic glycolysis
}

\author{
Chunjie Tian', Yeon Ju Kim², Sai Hali ${ }^{3}$, Oak-Sung Choo ${ }^{2,4}$, Jin-Sol Lee ${ }^{2,5}$, Seo-Kyung Jung ${ }^{2,5}$, Youn-Uk Choi ${ }^{6}$, \\ Chan Bae Park ${ }^{6}$ and Yun-Hoon Choung $\mathbb{1}^{24,5}$
}

\begin{abstract}
Age-dependent decrease of mitochondrial energy production and cellular redox imbalance play significant roles in age-related hearing loss (ARHL). Lactate dehydrogenase $B(L D H B)$ is a key glycolytic enzyme that catalyzes the interconversion of pyruvate and lactate. LDH activity and isoenzyme patterns are known to be changed with aging, but the role of LDHB in ARHL has not been studied yet. Here, we found that LDHB knockout mice showed hearing loss at high frequencies, which is the typical feature of ARHL. LDHB knockdown caused downregulation of mitochondrial functions in auditory cell line, University of Bristol/organ of Corti 1 (UB/OC1) with decreased $N^{\prime} A D^{+}$and increased hypoxia inducing factor-1a. LDHB knockdown also enhanced the death of UB/OC1 cells with ototoxic gentamicin treatment. On the contrary, the induction of LDHB expression caused enhanced mitochondrial functions, including changes in mitochondrial respiratory subunits, mitochondrial membrane potentials, ATP, and the NAD ${ }^{+} / \mathrm{NADH}$ ratio. Thus, we concluded that suppression of LDHB activity may be closely related with the early onset or progression of $\mathrm{ARHL}$.
\end{abstract}

\section{Introduction}

Age-related hearing loss (ARHL) that occurs in response to aging is a universal disorder in modern society. ARHL is characterized by an age-associated defect in hearing function, which begins with an increased hearing threshold in the high-frequency region and spreads toward the low-frequency region. Histologically, this process is characterized by the loss of hair cells and spiral ganglion neurons (SGNs) in the cochlea with age $^{1-3}$. The process of ARHL depends on many molecular, physiological, and biochemical changes ${ }^{4}$. Although the exact mechanism remained unclear, ARHL is caused by cumulative effect of extrinsic and intrinsic factors, such

\footnotetext{
Correspondence: Chan Bae Park (pcbkaist@gmail.com) or

Yun-Hoon Choung (yhc@ajou.ac.kr)

'Department of Otolaryngology, Dali Bai Autonomous Prefecture People's Hospital, Dali, Yunnan 671000, China

${ }^{2}$ Department of Otolaryngology, Ajou University School of Medicine, Suwon 16499, Republic of Korea

Full list of author information is available at the end of the article

These authors contributed equally: Chunjie Tian, Yeon Ju Kim, Sai Hali

Edited by G. Melino
}

as hereditary susceptibility, autophagic stress, inflammation, and oxidative stress ${ }^{5}$.

Decreased mitochondrial function with age has been documented in multiple mammalian species. Studies on mitochondria isolated from human muscle biopsies or rodent muscles support the existence of an intrinsic, aging-dependent mitochondrial defect associated with adenosine triphosphate (ATP) production ${ }^{6}$. Mitochondria are the main source of ATP and a direct intrinsic byproduct of this ATP production is reactive oxygen species (ROS). Under normal conditions, ROS are easily scavenged by various endogenous antioxidant enzymes activities, such as superoxide dismutase (MnSOD), catalase (CAT), and glutathione peroxidise (GPX). However, during aging, damaged mitochondria induced less ATP production and aberrant ROS generation, leading to oxidative stress and cellular senescence ${ }^{7}$.

Pyruvate is the substrate used for mitochondrial ATP production and is the product of the final step of glycolysis. In the presence of oxygen, pyruvate is further metabolized in the citric acid cycle to produce NADH and $\mathrm{FADH}_{2}$ for

\section{(c) The Author(s) 2020}

(c) (i) Open Access This article is licensed under a Creative Commons Attribution 4.0 International License, which permits use, sharing, adaptation, distribution and reproduction in any medium or format, as long as you give appropriate credit to the original author(s) and the source, provide a link to the Creative Commons license, and indicate if changes were made. The images or other third party material in this article are included in the article's Creative Commons license, unless indicated otherwise in a credit line to the material. If material is not included in the article's Creative Commons license and your intended use is not permitted by statutory regulation or exceeds the permitted use, you will need to obtain permission directly from the copyright holder. To view a copy of this license, visit http://creativecommons.org/licenses/by/4.0/. 
oxidative phosphorylation in the mitochondrion ${ }^{8}$. Lactate dehydrogenase (LDH) catalyzes the interconversion of pyruvate and lactate using nicotinamide adenine dinucleotide $\left(\mathrm{NAD}^{+}\right)$and $\mathrm{NADH}$ as a cofactor during the last step of glycolysis. LDH is a homo- or hetero-tetrameric enzyme composed of two subunits, $M$ (muscle) and $H$ (heart), which are encoded by the LDHA and LDHB genes, respectively ${ }^{9}$. Five different isozymes can be formed depending on the ratio of the $\mathrm{M}$ and $\mathrm{H}$ subunits. The major isozyme in skeletal muscle and liver, with four "M" LDHA subunits, is called LDH5 and mainly contributes to the generation of lactate from pyruvate. Meanwhile, the major isozyme in heart muscles, known as LDH1, contains four heart " $H$ " LDHB subunits and functions in the production of pyruvate from lactate. Isozymes that contain more LDHA subunits will generally undergo reactions to produce lactate from pyruvate, whereas isozymes with more LDHB react to produce pyruvate from lactate. Other variants contain both types of subunits (M3H1:LDH4, M2H2:LDH3, and M1H4: $\mathrm{LDH} 2)^{10}$. The organ of Corti in the inner ear contains LDH1 (LDHB) rather than LDH5 (LDHA) due to its highenergy requirements ${ }^{11}$.

Conversion from lactate to pyruvate depends on an LDHB-containing isozyme, which is important in maintaining a high level of pyruvate and is highly expressed in tissues with high-energy demands. If the conversion from lactate to pyruvate is disrupted, the substrate concentration for mitochondrial ATP production will decrease, resulting in mitochondrial dysfunction ${ }^{12}$.
In this regard, one study showed that a decreased level of nuclear $\mathrm{NAD}^{+}$contribute to mitochondria defects during skeletal muscle aging in a $\mathrm{NAD}^{+}$-dependent deacetylase, sirtuin 1 (SIRT1)-dependent manner. Moreover, calorie restriction slowed aging process by raising $\mathrm{NAD}^{+}$ and SIRT $1^{13}$. In addition, augmentation of $\mathrm{NAD}^{+}$by $\beta$-lapachone, a known modulator of cellular $\mathrm{NAD}^{+}$by conversion of $\mathrm{NADH}$ to $\mathrm{NAD}^{+}$, effectively prevented ARHL through the reducing inflammation and oxidative stress, mitochondrial damage in rodents ${ }^{14}$. However, the functional significance of the LDHB in ARHL is unclear. Therefore, the purposes of this study were to elucidate the metabolic changes in ARHL using LDHB knockout (KO) mice and to determine the significance of LDHB in the process of ARHL.

\section{Results}

Generation of whole-body LDHB KO mice

To create LDHB gene knockout mouse strains, we first generated LDHB floxed (LDHB loxP/+) mice using homologous recombination in mouse embryonic stem (ES) cells (Fig. 1a). Southern blot analysis revealed ES cells with the target LDHB locus (Fig. 1b). We generated wholebody LDHB gene $\mathrm{KO}$ mice by crossing LDHB floxed (LDHB loxP/+) mice with protamine-cre (PRM-cre) mice, which express Cre recombinase in sperm. The resulting double heterozygous mice (LDHB +/loxP, +/PRM-cre) were mated with wild-type (WT) mice to generate heterozygote whole-body LDHB knockout (LDHB +/-)
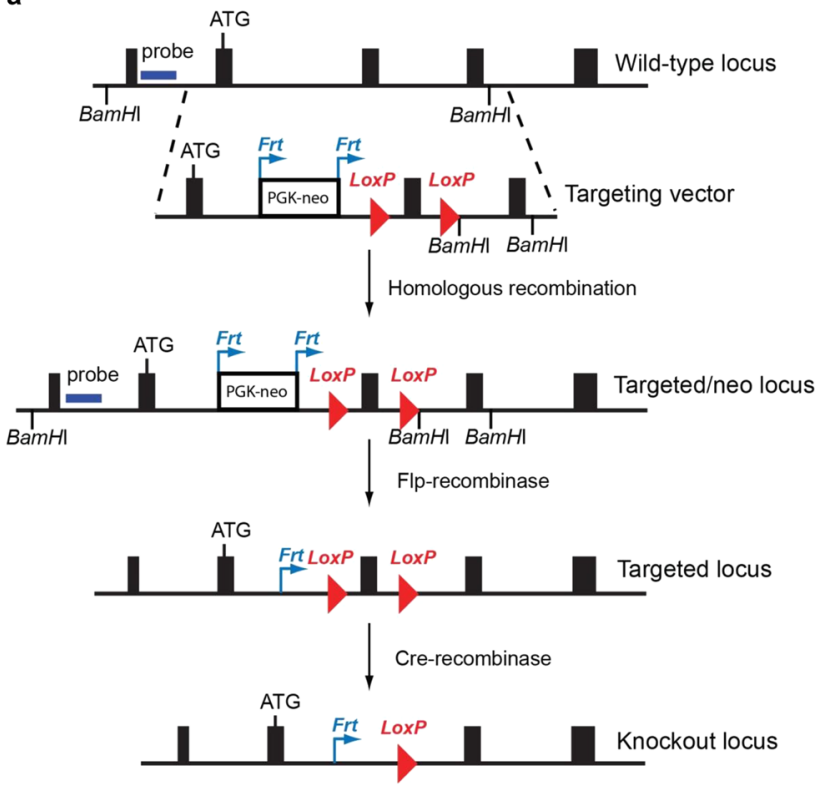

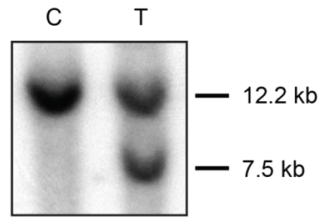

C

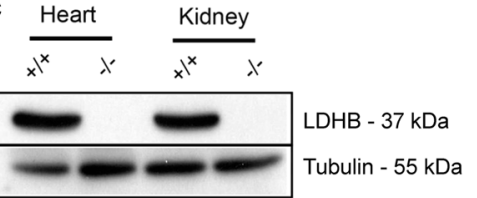

d

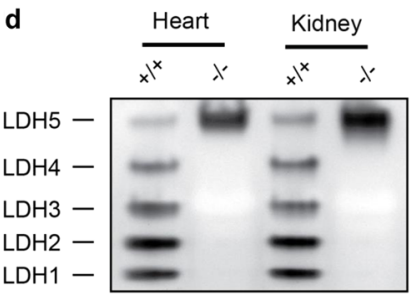

Fig. 1 Generation of LDHB KO mice. a Targeting of the LDHB gene was performed by floxing exon 3 of the LDHB gene with the PGK-neo cassette containing Frt sites. $\mathbf{b}$ Screening of target ES cells for LDHB with the probe indicated in Fig. 1a. Control (c) and target (T) ES cells. $\mathbf{c}$, $\mathbf{d}$ Confirmation of LDHB knockout in the heart and kidney through western blot analysis (c) and gel LDH isomer activity assay (d). 
mice, and homozygote LDHB knockout (LDHB -/-) mice were generated by crossing the heterozygote LDHB knockout (LDHB +/-) mice. The generation of wholebody homozygote LDHB (LDHB -/-) mice was confirmed based on complete deficiency of LDHB protein (Fig. 1c) and LDH 1-4 isozymes (Fig. 1d) in the heart and kidney of each $\mathrm{KO}$ mouse. In the cochlea, LDHB was broadly expressed throughout the organ of Corti, spiral limbus, spiral ganglion, and spiral ligament, and LDHB KO mice did not contain detectable LDHB protein (Supplementary Fig. 1).

\section{LDH activity and body weight growth of LDHB KO mice}

LDHB is important for maintaining a high level of pyruvate and is highly expressed in tissues with highenergy demands, such as the heart and kidney. In response to $\mathrm{KO}, \mathrm{LDH}$ activity from the lactate side of the reaction (lactate to pyruvate: $\mathrm{L}-\mathrm{P}$ ) showed a prominent decrease due to deficiency of LDHB in terms of total activity. $\mathrm{LDH}$ activity on the pyruvate side of the reaction (pyruvate to lactate: $\mathrm{P}-\mathrm{L}$ ) showed a slight decrease in total LDH activity as well. As the LDHA protein level did not change in LDHB KO mice, total LDH decreased with LDHB deficiency (Fig. 2a). To determine whether loss of the LDHB gene affects the growth of mice, the body weights of male and female groups of LDHB KO mice were measured for 48 weeks. The LDHB KO mice showed normal growth (Fig. 2b).

\section{Hearing levels and cochlear morphologic findings of LDHB KO mice during aging}

Hearing function was quantified using the auditory brainstem response (ABR) test in WT and LDHB KO mice at 2, 5, and 10 months of age. Both WT and LDHB $\mathrm{KO}$ mice showed normal hearing thresholds at 8,16 , and $32 \mathrm{kHz}$ frequencies at the age of 2 months (Fig. 3a). WT mice at 5 months had normal hearing thresholds across frequencies $(10.0 \pm 0.0 \mathrm{~dB} / 12.0 \pm 2.6 \mathrm{~dB} / 13.0 \pm 4.2 \mathrm{~dB}$ at $8 /$ $16 / 32 \mathrm{kHz}$, respectively). By contrast, LDHB KO mice exhibited an early onset of progressive hearing loss, with their mean thresholds increasing to $15.0 \pm 5.3 \mathrm{~dB} / 24.5 \pm$ $8.0 \mathrm{~dB} / 28.0 \pm 9.2 \mathrm{~dB}$ at $8 / 16 / 32 \mathrm{kHz}$, respectively. The differences between the two groups were statistically significant at $16 \mathrm{kHz}(P<0.05)$ and $32 \mathrm{kHz}(P<0.01)$ (Fig. $3 a)$. By 10 months of age, ARHL developed at high frequencies in the WT mice, with increased hearing thresholds of $15.0 \pm 3.3 \mathrm{~dB} / 26.0 \pm 4.0 \mathrm{~dB} / 30.0 \pm 3.3 \mathrm{~dB}$ at $8 / 16 / 32 \mathrm{kHz}$. The LDHB-KO mice showed worsening of hearing thresholds, reaching $18.0 \pm 2.6 \mathrm{~dB} / 39.0 \pm 4.6 \mathrm{~dB} /$ $43.0 \pm 4.8 \mathrm{~dB}$ at $8 / 16 / 32 \mathrm{kHz}$. Hearing ability decreased for high-frequency sound, while the low-frequency area was preserved. At the age of 10 months, LDHB KO mice showed more severe hearing loss than WT mice. It is widely known that the onset of ARHL begins with high frequencies and spreads toward lower frequency regions during aging ${ }^{15}$. The differences in hearing thresholds between the two groups were statistically significant at $16 \mathrm{kHz}(P<0.01)$ and $32 \mathrm{kHz}(P<0.01)$ (Fig. $3 \mathrm{a})$.

Scanning electron microscopy (SEM) findings showed that the presence of stereocilia on hair cells did not significantly differ between WT and LDHB KO mice at 2 months (Fig. 3b), but the number of stereocilia in the middle and basal turns was reduced at 5 months of age in KO mice. Consistent with ABR results indicating higher hearing thresholds at $32 \mathrm{kHz}$, samples of high-frequency areas showed fewer remaining hair cells than those at $16 \mathrm{kHz}$. The difference in the number of hair cells was statistically significant between the two groups both at $16 \mathrm{kHz}(P<0.01)$ and $32 \mathrm{kHz}(P<0.01)$ (Fig. 3b). Hair cell loss was detected in 10-month-old WT mice in the middle and basal turns, but not in the apical turn. By contrast, 10-month-old KO mice had hair cell loss across
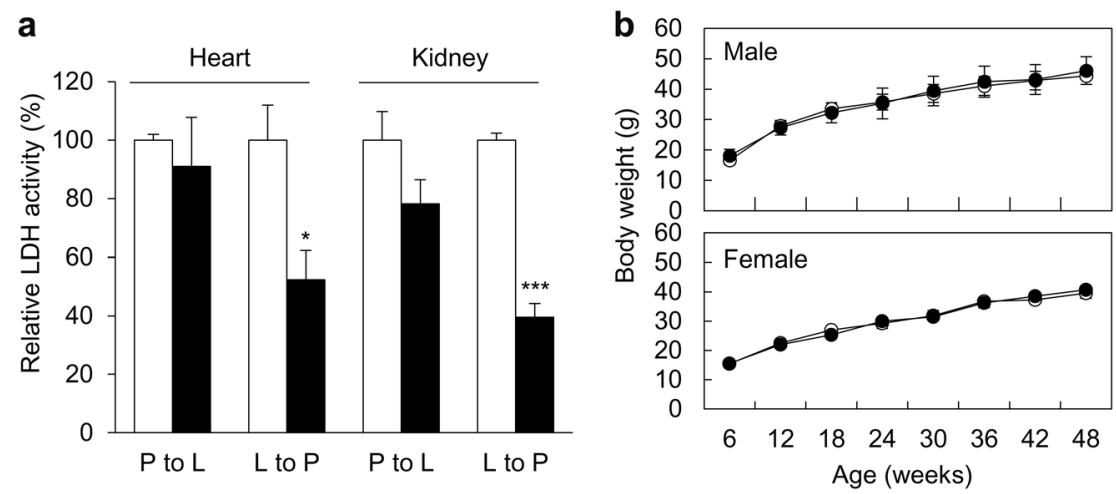

Fig. 2 LDH activity and body weight growth of LDHB KO mice. LDH activities decreased in the heart and kidney of LDHB KO mice (a). The body weight of LDHB KO mice increased normally (b). White bars and circles represent WT mice, and black bars and circles represent $K O$ mice. ${ }^{*} P<0.05$, ${ }^{*} P<0.01,{ }^{* *} P<0.001$ vs. WT with Student's $t$-test. 

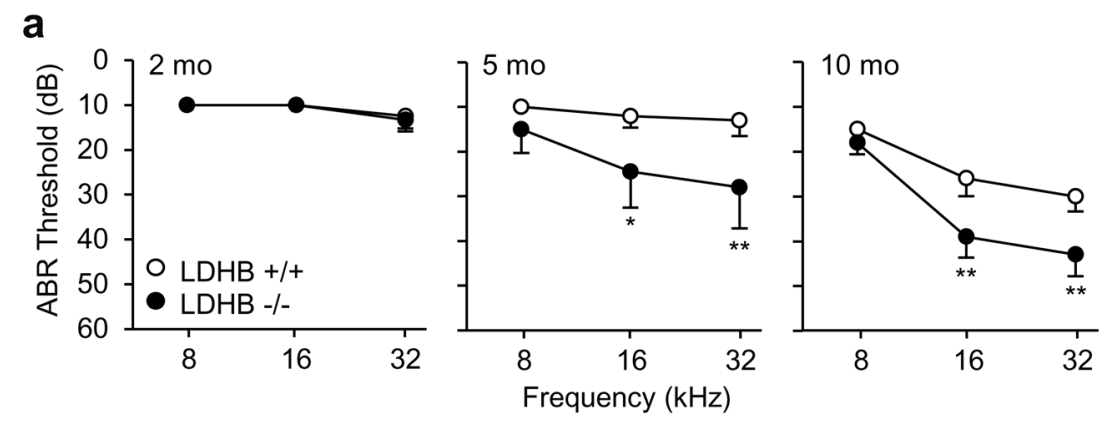

b

Distance from apex (\%)
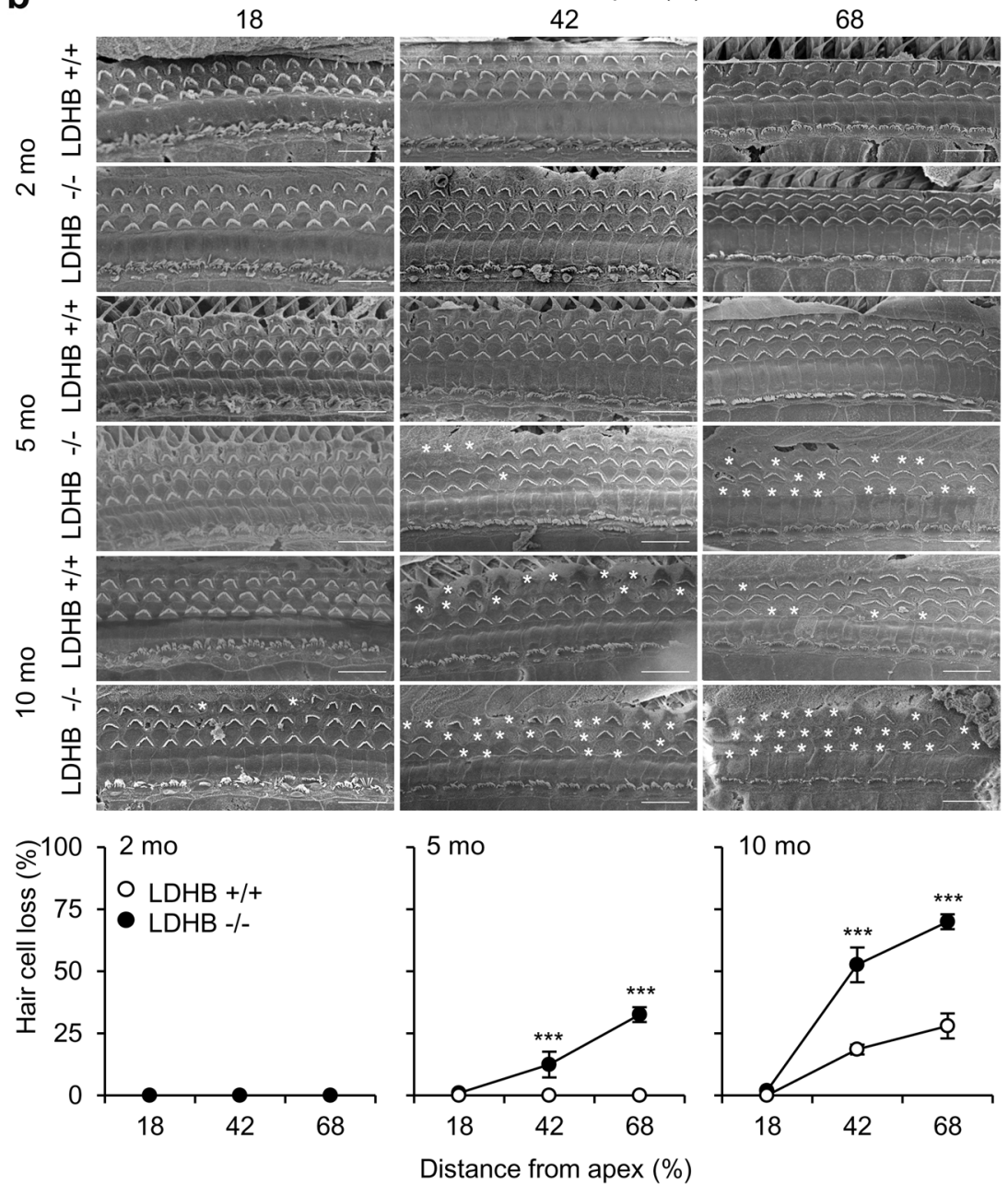

Fig. 3 Hearing levels and cochlear morphologic findings of LDHB KO mice during aging. a Increased ABR thresholds were observed in LDHB $\mathrm{KO}$ mice $(-/-$, black circle) at the age of 5 months and in WT mice (+/+, white circle) at 10 months. LDHB KO mice showed more severe worsening of hearing than WT mice. $\mathbf{b}$ SEM findings showed that the number of stereocilia was reduced in the middle (42\% distance from apex) and basal turns (68\% distance form apex) at 5 and 10 months of age in KO mice and only at 10 months in the WT mice. The difference in hair cell death was statistically significant between the LDHB KO and WT groups of mice. Scale bar $=10 \mathrm{~nm}$. ${ }^{*} P<0.05,{ }^{* *} P<0.01,{ }^{* * *} P<0.001$ vs. WT with Student's t-test.

all turns, including the apical turn. Closer to the base, fewer hair cells remained. The numbers of hair cells in 10month-old KO mice were lower than those in 10-monthold WT mice in all turns, and statistical significance was detected in the middle and basal turns $(P<0.01)$ (Fig. 3b). Cochlear surface preparation at three different age points (2, 5, and 10 months) across frequencies indicated no inner hair cell loss for either group of animals (Fig. 3b). 


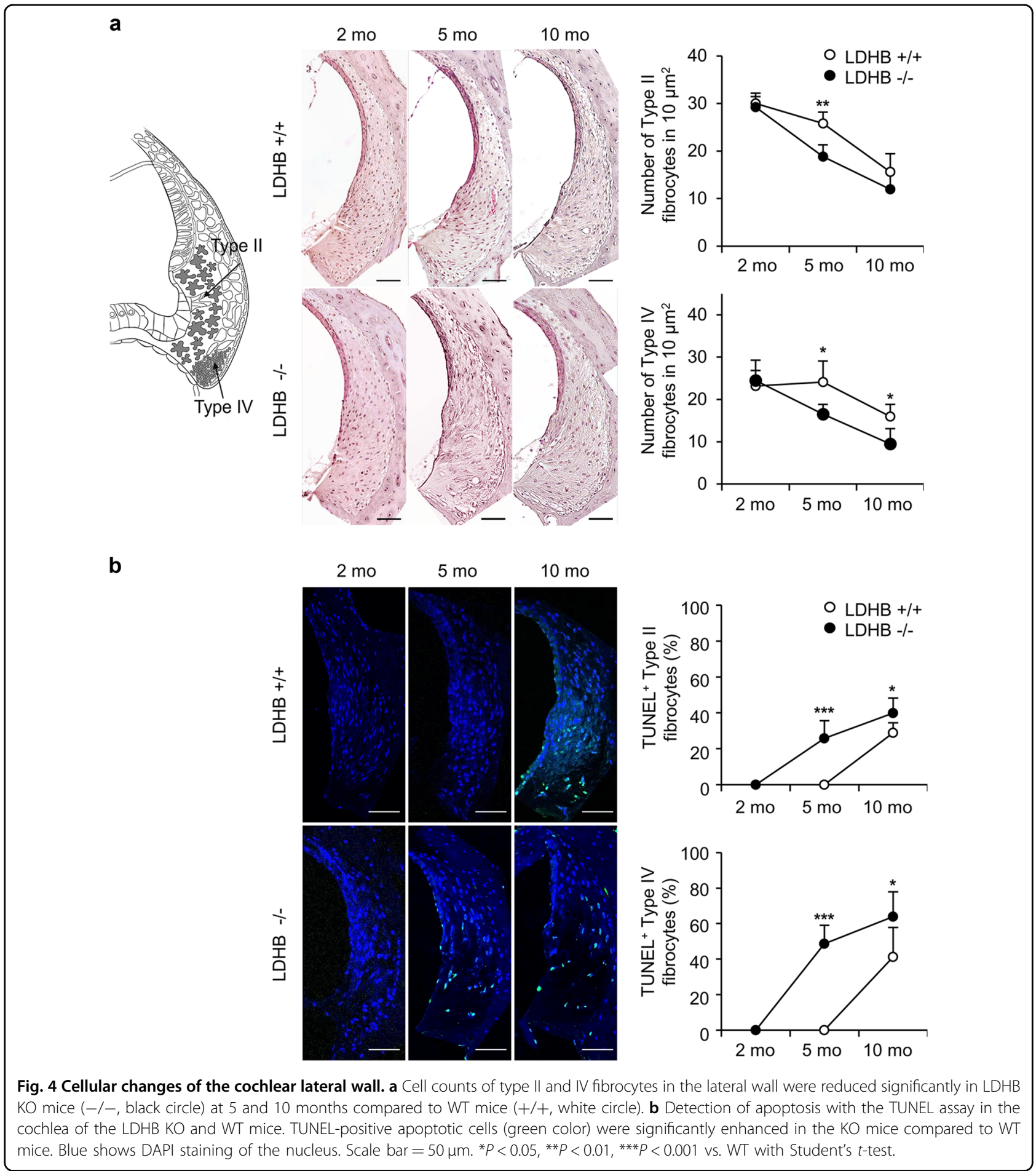

\section{Cellular change of cochlear lateral walls}

Different types of fibrocytes are shown in a diagram of the lateral wall (Fig. 4a, left). Compared with findings from 2-month-old WT mice, fibrocytes in the lateral wall showed no changes in 5-month-old WT mice, while diffuse degeneration of type II and IV fibrocytes was detected in 5-month-old LDHB KO mice. Comparison of mean cell counts (cells $/ 10.000 \mu \mathrm{m}^{2}$ ) in the type II and type IV areas revealed statistically significant differences in both groups at the age of 5 months (Fig. 4a).

The terminal deoxynucleotidyl transferase dUTP nick end labeling (TUNEL) assay was applied to identify cellular apoptosis in the cochlear lateral wall. No differences were observed between 2-month-old WT and KO mice, 


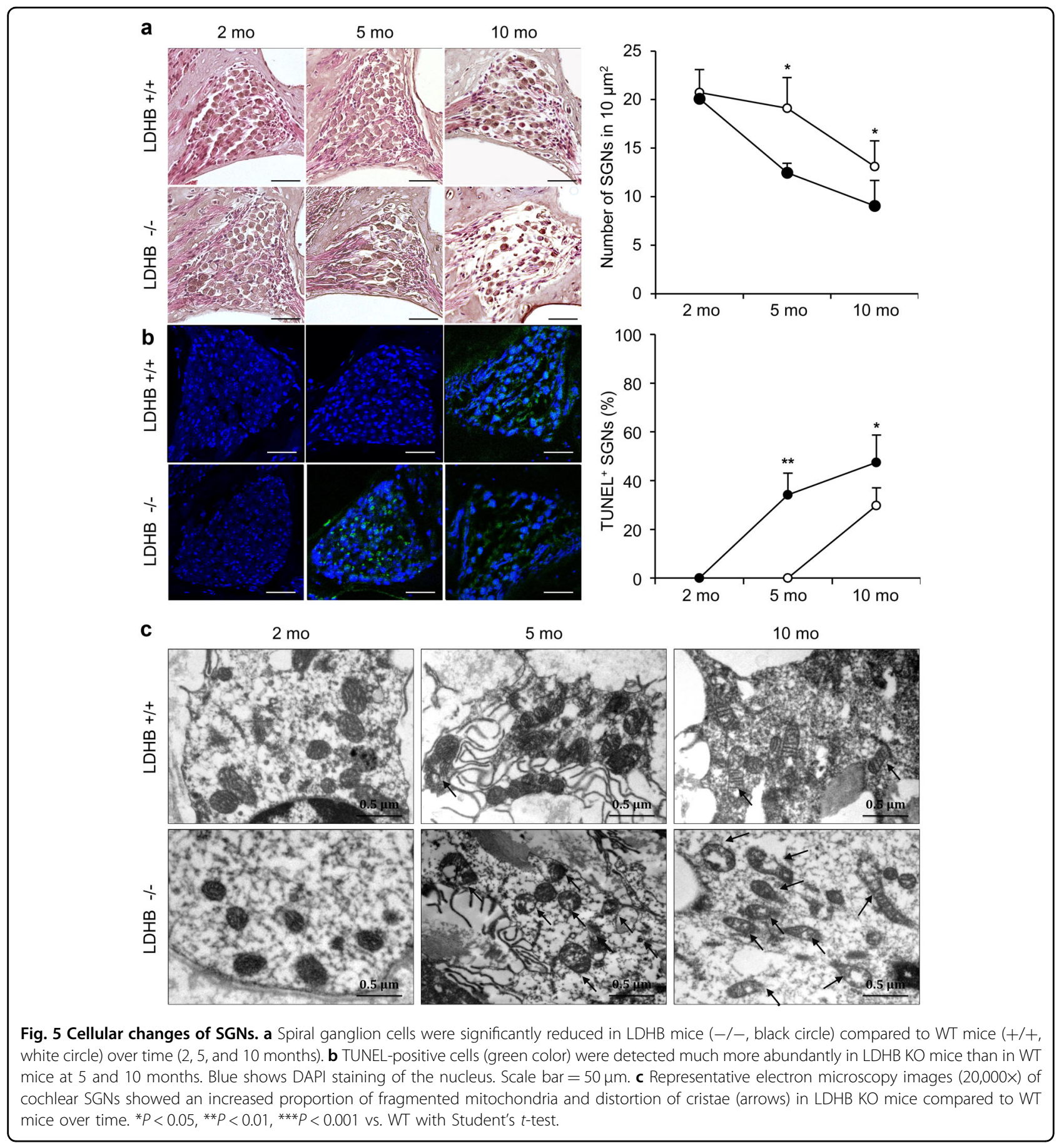

while TUNEL-positive fibrocytes were detected in 5month-old $\mathrm{KO}$ mice and increased at the age of 10 months. The difference in TUNEL-positive cell expression was significant between the two groups $(P<$ 0.01 at 5 months and $P<0.05$ at 10 months) (Fig. $4 \mathrm{~b}$ ).

\section{Cellular changes of SGNs}

Progressive loss of SGNs through the aging process was detected in the $\mathrm{KO}$ mice at the ages of 5 and 10 months, and was observed at 10 months in WT mice. Cell counts (cells/10.000 $\mathrm{m}^{2}$ ) of SGNs showed statistical significance at the ages of 5 and 10 months (Fig. 5a).

TUNEL-positive spiral ganglion cells were observed in 5-month-old $\mathrm{KO}$ mice and increased in 10-month-old $\mathrm{KO}$ mice, whereas WT mice showed TUNEL-positive expression only at the age of 10 months, not at 5 months. Differences in the ratio of TUNEL-positive cells to total cells were statistically significant (Fig. 5b). 
Considering the essential role of LDHB in mitochondrial function ${ }^{16}$, we evaluated whether there were alterations in mitochondrial morphology in LDHB KO mice through transmission electron microscopy (TEM) analysis. An increased proportion of fragmented mitochondria and distortion of cristae were observed in spiral ganglion cells at the age of 10 months in both WT and KO mice. LDHB KO mice showed more severe mitochondrial fragmentation than WT mice (Fig. 5c).

\section{LDHB levels and mitochondrial function in differentiated UB/OC1 cells}

To investigate the molecular mechanism of hair cell loss in LDHB KO mice in the present study, UB/OC1 cells were used. This cell line can differentiate into hair cells under the conditions of $37{ }^{\circ} \mathrm{C}$ and $5 \% \mathrm{CO}_{2}{ }^{17}$. Under differentiation conditions, the hair cell marker Myosin VIIa was upregulated and a supporting cell marker, Jagged 1, was downregulated in differentiated UB/OC1 cells (Fig. 6a). A clear increase of LDHB was observed in differentiated UB/OC1 cells (Fig. 6a, b). By contrast, there was no prominent change in LDHA expression compared with undifferentiated UB/OC1 cells.

The effect of LDHB induction on mitochondrial function was evaluated using two critical indicators of energy status: intracellular ATP and $\mathrm{NAD}^{+} / \mathrm{NADH}$ levels. An increased level of extracellular ATP was observed in differentiated UB/OC1 cells (Fig. 6c). Moreover, increases in the expression of mitochondrial respiratory proteins, mitochondrial RNA content, and mitochondrial membrane potential (MMP) were observed compared to undifferentiated UB/OC1 cells (Fig. 6d, f).

Additionally, $\mathrm{NAD}^{+} / \mathrm{NADH}$ levels, ROS generation, LDH activity, and lactate production were measured (Data not shown). With an increase in mitochondrial respiratory chain activity, the electron donor NADH was significantly converted to $\mathrm{NAD}^{+}$, resulting in increases in redox status and $\mathrm{NAD}^{+} / \mathrm{NADH}$ level in differentiated $\mathrm{UB} /$ OC1 cells. Mitochondria are known to play a critical role in aerobic ATP generation and are the primary source of ROS for various pathways. We found that intracellular ROS production was upregulated in differentiated UB/ OC1 cells. In addition, differentiated UB/OC1 cells had higher LDH activity and accordingly released a larger amount of lactate into the medium than undifferentiated UB/OC1 cells (Supplementary Fig. 2). These results suggest that differentiated $\mathrm{UB} / \mathrm{OC} 1$ cells upregulated $\mathrm{LDHB}$ expression and exhibit increased mitochondrial function.

\section{Effect of LDHB knockdown on mitochondrial functions and ototoxic cell death}

To investigate the mechanistic relationship between hair cell loss and LDHB, we evaluated mitochondrial functional changes using LDHB small-interfering RNA (siRNA) in differentiated UB/OC1 cells. As shown in Fig. 7a, LDHB protein expression decreased after knockdown, a

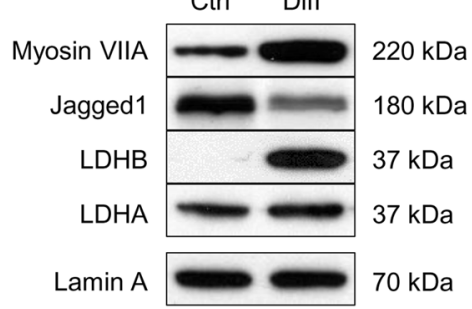

d

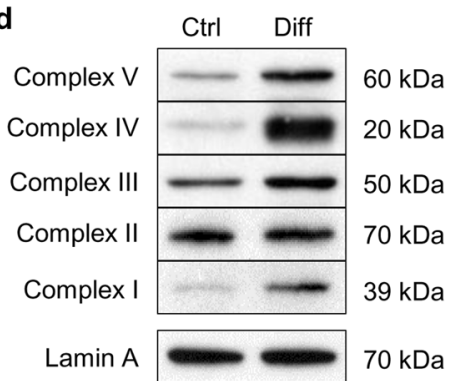

b

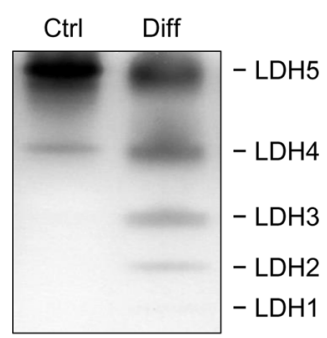

e

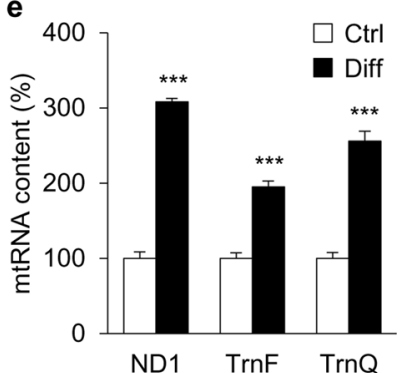

c

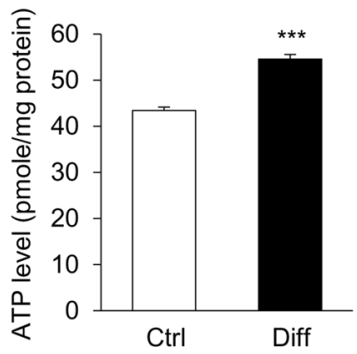

f

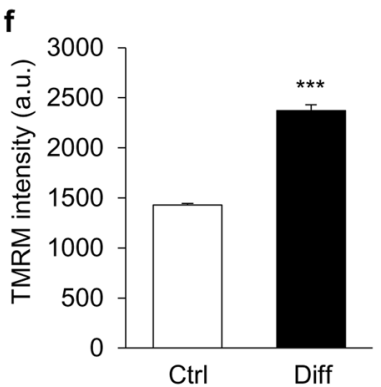

Fig. $6 \mathrm{LDHB}$ levels and mitochondrial function of differentiated UB/OC1 cells. UB/OC1 cells were cultured under permissive conditions of $37^{\circ} \mathrm{C}$ and $5 \% \mathrm{CO}_{2}$ without $\gamma$-interferon to allow differentiation into hair cells. Expression of LDHB (a), ATP level (c), mitochondrial respiratory subunits (d), mitochondrial RNA levels (e), and MMP (f) increased in differentiated UB/OC1 cells compared to control cells. $\mathbf{b}$ The isozyme pattern shifted toward LDH2 in differentiated UB/OC1 cells. White bars represent undifferentiated control cells (Ctrl) and black bars represent differentiated cells (Diff). ${ }^{*} P<$ $0.05,{ }^{* *} P<0.01,{ }^{* * *} P<0.001$ vs. undifferentiated cells with Student's t-test. 


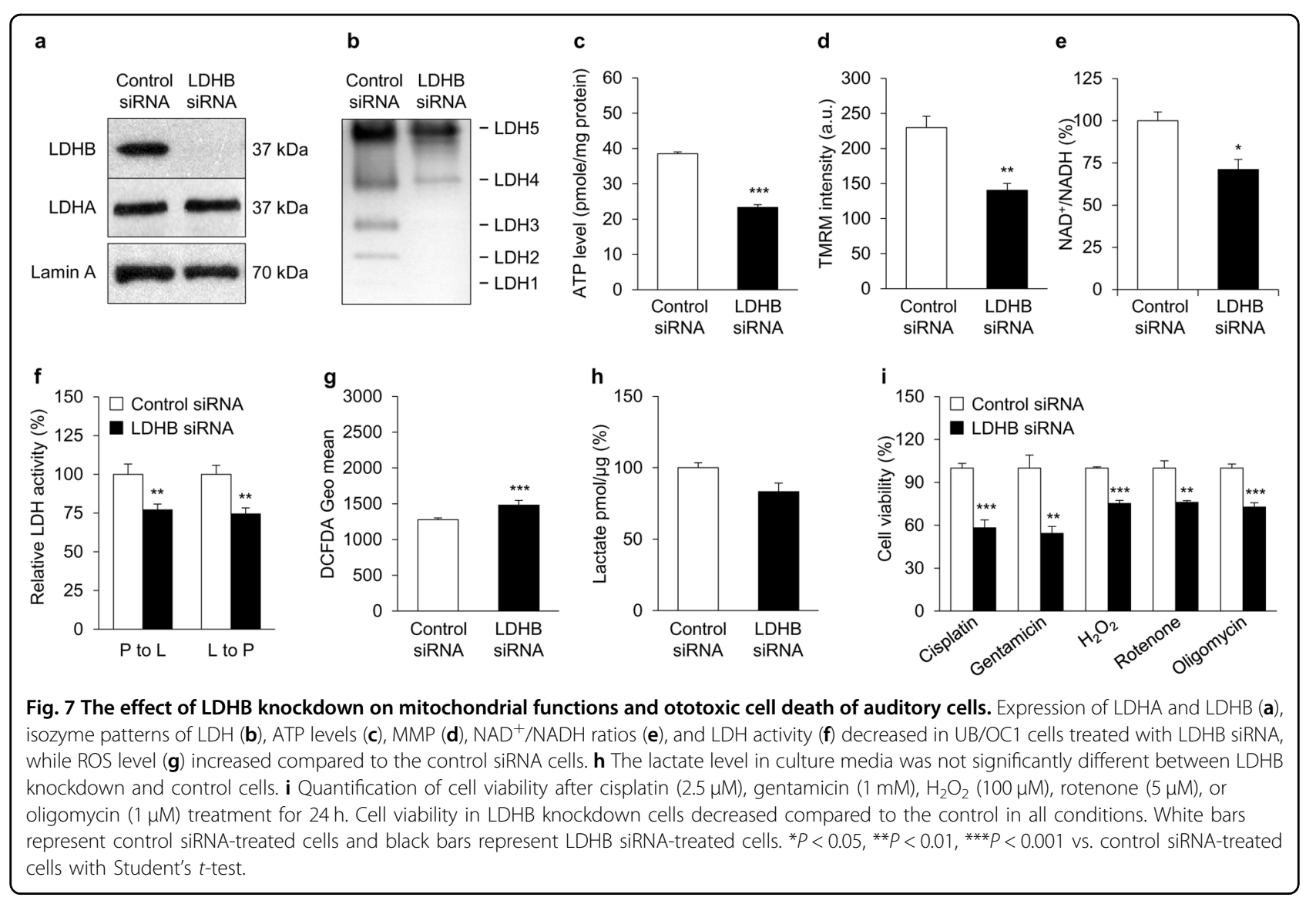

without any change in LDHA expression. The LDH isozyme pattern changed from a mix of five different patterns to favor the LDH5- and LDH4-containing patterns (Fig. 7b). Knockdown of LDHB led to downregulation of ATP production (Fig. 7c), MMP (Fig. 7d), and the $\mathrm{NAD}^{+} /$ $\mathrm{NADH}$ ratio (Fig. 7e). Relative LDH activity decreased in both the P-L and L-P reactions in LDHB siRNA-treated UB/OC1 cells (Fig. 7f). Additionally, LDHB knockdown cells significantly upregulated ROS production compared to control siRNA cells (Fig. 7g). On the other hand, there was no clear change in the amount of lactate released into the medium compared with control siRNA-treated cells (Fig. 7h). Collectively, these results indicate that LDHB knockdown decreased mitochondrial function in differentiated UB/OC1 cells.

Mitochondrial DNA mutations/deletions and excessive ROS production cause mitochondrial dysfunctions, which may be essential mechanisms of age-related auditory disorders ${ }^{18}$. To investigate the sensitivity of LDHBdeficient auditory cells to mitochondrial toxins, a mitochondrial respiratory chain inhibitor, such as rotenone (complex I inhibitor), carbonyl cyanide m-chlorophenyl hydrazone (CCCP; mitochondrial uncoupling agent), antimycin A (complex III inhibitor), or oligomycin (complex $\mathrm{V}$ inhibitor) was used. LDHB knockdown significantly increased cell death of UB/OC1 cells compared to control siRNA-treated cells under $\mathrm{H}_{2} \mathrm{O}_{2}$, rotenone, and oligomycin treatments (Fig. 7i). Cisplatin and gentamicin are well-known ototoxic drugs, and auditory cell death is mediated by the generation of $\operatorname{ROS}^{19}$. Cisplatin accumulates in mitochondria, causing direct and significant impairment of mitochondrial DNA (mtDNA) and $\mathrm{mtRNA}^{20}$. LDHB deficiency significantly increased the sensitivity of UB/OC1 cells to cisplatin and gentamicin (Fig. 7i). These results show that depletion of LDHB in mitochondria makes differentiated auditory cells much more vulnerable to oxidative stress and damage from ROS originating in the mitochondria, and suggest that downregulation of $\mathrm{LDHB}$ is a potential contributor to the progression of ARHL.

\section{Discussion}

Mitochondrial pathology plays an important role in different types of hearing loss ${ }^{21}$. Acquired mitochondrial dysfunction in hearing loss, including mtDNA mutation, mitochondrial turnover, and apoptosis, appears to involve increased ROS and decreased energy production, as well as redox imbalance ${ }^{22}$. Tissues that consist of post-mitotic cells, such as brain and inner ear cells, are particularly vulnerable to mitochondrial dysfunction due to their 
high-energy requirements and inability to undergo regeneration. It is well-known that pyruvate is the substrate of mitochondrial ATP production and final product of glycolysis. LDHB is important for maintaining a high pyruvate level and thus LDHB expression could be directly related to mitochondrial function and energy production. A widely accepted hypothesis for the possible cause of ARHL is mitochondrial dysfunction related to ROS, mtDNA mutation, and ATP deficiency ${ }^{23}$.

In the present study, we generated whole-body LDHB gene $\mathrm{KO}$ (LDHB $-/-$ ) mice by crossing heterozygous LDHB knockout (LDHB $+/-$ ) mice. LDHB KO mice showed a decrease in total LDH activity in both the lactate side of the reaction (lactate to pyruvate: L-P) and the pyruvate side (pyruvate to lactate: $\mathrm{P}-\mathrm{L}$ ). Based on previous research $^{24}$ and our experimental observations, LDHB is highly sensitive to substrate inhibition. At higher concentrations of pyruvate, LDHB activity is inhibited. In our experiments, we selected $0.5 \mathrm{mM}$ pyruvate as the substrate to avoid substrate inhibition. In addition, the LDHA protein level was not affected in LDHB KO mice, and therefore total LDH activity also decreased due to LDHB deficiency on the pyruvate side of the reaction, which is consistent with cell line study results (Fig. 7).

In this study, we have shown for the first time that deficiency of the LDHB gene caused early onset of ARHL using LDHB KO mice. LDHB KO mice showed aggravation of ARHL with an increased number of dysfunctional mitochondria and loss of auditory cells. The hearing threshold shift observed in aging WT mice was similar to a previous description of C57/BL6 mice ${ }^{4}$, whereas LDHB $\mathrm{KO}$ mice exhibited more rapid worsening of hearing thresholds at 5 and 10 months of age especially in the high-frequency area, which is characteristic of ARHL. ARHL is mainly rooted in the pathology of cochlear cells, including hair cells, SGNs, and lateral wall fibrocytes. At 5 and 10 months of age, LDHB KO mice displayed earlier and more severe degeneration of hair cells, SGN death, and loss of type II and IV fibrocytes in the lateral wall, which all accompanied by mitochondrial damage. These results indicated that deficiency of LDHB might accelerate degeneration of cochlear cells in the early stage of senescence. However, we cannot exclude the possible contribution of other organs, such as the brain, to the failure of the auditory system in LDHB KO mice. The decline in brain function caused by LDHB deficiency and its impact on ARHL development are under study.

The relationship between LDHB and ARHL is not wellknown, but some studies have shown that LDHB activity decreases during the aging process, leading to age-related diseases. Aging WT mice and prematurely aging mtDNA mutator mice had significantly increased LDHA/LDHB activity ratios, which led to elevated lactate levels in the brain $^{25,26}$. Decreased LDHA and LDHB activity has also been demonstrated to be predictive of aging phenotypes in muscle ${ }^{27}$. Regulation of LDH plays a key role in regulating aerobic glycolysis through conversion of pyruvate to lactate through coupled oxidation of $\mathrm{NADH}$ to $\mathrm{NAD}^{+28}$. Recently, decreased intracellular $\mathrm{NAD}^{+} / \mathrm{NADH}$ ratios have been reported in aged tissues. A decrease in the $\mathrm{NAD}^{+} / \mathrm{NADH}$ ratio leads to poly (ADP-ribose) polymerase 1 (PARP1) hyper-activation via altered redox mechanisms and consequent DNA damage ${ }^{29}$. Furthermore, SIRT1 activity and $\alpha$-ketoglutarate dehydrogenase $(\alpha-K G D H)$ complex, an enzyme complex of the citric acid cycle the ROS generation, are influenced by the $\mathrm{NAD}^{+}$/ NADH ratio. Recently, PARP1 was identified as an important target in oxidative stress-induced cochlear marginal cell death modulating parthanatos and autophagy $^{30}$. Moreover, some reports have suggested that SIRT1 is abundantly expressed in young cochlear cells and that SIRT1 deficiency may be associated with $\mathrm{ARHL}^{31}$. Therefore, the intracellular $\mathrm{NAD}^{+} / \mathrm{NADH}$ ratio may be a critical downstream target in LDHB KO mice, leading to progressive loss of hearing.

We found that an increase of LDHB caused enhancement of mitochondrial function with increases in $\mathrm{NAD}^{+} / \mathrm{NADH}$ balance, ATP level, and mitochondrial respiratory chain subunits observed in differentiated UB/OC1 cells (Fig. 6). The mitochondrial respiratory chain catalyzes NADH oxidation. Increased mitochondrial respiratory chain activity elevates the $\mathrm{NAD}^{+} /$ NADH ratio and causes translocation of protons across the inner mitochondrial membrane, which ultimately leads to increased ATP production. Knockdown of LDHB caused mitochondrial dysfunction and triggered a decline in the $\mathrm{NAD}^{+} / \mathrm{NADH}$ ratio (Fig. 7). This decrease in the $\mathrm{NAD}^{+} / \mathrm{NADH}$ ratio likely caused mitochondrial dysfunction in UB/OC1 cells.

The increased sensitivity of LDHB knockdown cells to mitochondrial toxins or ototoxic drugs observed in the present study might be caused by mitochondrial dysfunction. Cisplatin and gentamicin are commonly used drugs in the clinic. However, both of these drugs show ototoxicity as a side effect, inducing oxidative stress and mitochondrial defects in the cochlea of the inner ear ${ }^{19}$. The present study showed that LDHB deficiency resulted in mitochondrial defects in auditory cells, which increased sensitivity to ototoxic drugs such as cisplatin and gentamicin.

In conclusion, our findings provide evidence that deletion of LDHB leads to dysfunctional mitochondria, thereby accelerating ARHL. Furthermore, we demonstrated that MMP, ATP, and the $\mathrm{NAD}^{+} / \mathrm{NADH}$ ratio, which are related to mitochondrial function, are reduced in LDHB knockdown cells. These results suggest that LDHB may be used as a useful biomarker for predicting ARHL progression. 


\section{Materials and methods}

\section{Generation of LDHB knockout mouse}

The genomic locus of the LDHB gene was modified in mouse ES cells so that two loxP sites flanked exon 3 of the gene. To generate the targeting vector used for genomic modification, we first identified a bacterial artificial chromosome (BAC) clone containing the LDHB gene in a 129Sv RPCI-22M BAC library (Invitrogen) through hybridization with an LDHB cDNA probe. Then, a 10.5$\mathrm{kb}$ genomic fragment containing exons 2-4 of LDHB was excised from the BAC and cloned into pBluescript II $\mathrm{SK}+$ (Strategene) through the ET recombination technique to generate a plasmid, pBS-LDHB (Liu, Genome Res). To introduce a loxP site at the intron $3^{\prime}$ of exon 3 , an oligonucleotide containing the loxP site was inserted at the KpnI site of pBS-LDHB, and the resulting plasmid was designated pBS-LDHBLoxP. Finally, the Frt-PGK-neoFrt-loxP cassette (Frt-site-flanked neomycin resistance gene with loxP site located at its $3^{\prime}$ end) was inserted at the PacI site of pBS-LDHBLoxP to create the LDHB targeting vector, $\mathrm{pBS}$-LDHBKO. The PacI fragment of the Frt-PGK-neo-Frt-loxP cassette was excised from a modified pDELBOY-3X plasmid where PacI sites were introduced at the XhoI and ClaI site (Teglund, Cell). Next, the NotI-linearized LDHB targeting vector was electroporated into ES cells and those clones that grew in the presence of the antibiotic gentamycin were selected. Genomic DNA was isolated from gentamycin-resistant ES cell clones, digested with BamHI, and subjected to Southern blot analysis using a genomic DNA probe located 5 -upstream of the target sequence. Among 130 ES cell clones that were analyzed, two target clones were found. Chimeras were generated through blastocyst injection of target ES cells and germline transmission was achieved from both clones. The Frt-flanked neomycin resistance gene was excised by mating LDHB +/neo-loxP mice to transgenic mice ubiquitously expressing Flp-recombinase, thus generating $\mathrm{LDHB}+/$ loxP.

$\mathrm{LDHB}+/$ loxP mice were mated with transgenic mice expressing Cre recombinase under the control of the mouse protamine1 promoter (PRM-cre). Prm-cre mice express Cre recombinase during the terminal haploid stages of spermatogenesis. The resulting double heterozygous mice (LDHB+/loxP, +/PRM-cre) were mated with WT C57BL6 mice to generate heterozygote whole-body LDHB knockout (LDHB +/-) mice, and homozygote LDHB knockout (LDHB -/-) mice were generated by crossing heterozygote LDHB knockout (LDHB $+/-$ ) mice. All animal procedures were approved by the institutional review board of Ajou University School of Medicine (AJIRB-BMR-SMP-13-181). For all animal experiments, we used five age-matched animals selected randomly from cages of both WT and LDHB KO mice. Animals with abnormal behavior of with obvious rough coat were excluded to minimize individual variance. Researcher who were blinded to the all experimental groups.

\section{Auditory brainstem response (ABR) analysis}

ABR was tested with the Biosig 32 system (TuckerDavis Technologies, Gainesville, FL, USA), as described previously $^{31}$. Mice were anesthetized with an injection of Zoletil 50 (Virbac Laboratories, Carros, France) and 2\% Rompun (Bayer Korea, Ansan, Korea). Prior to placement of earphones, all tympanic membranes were examined with an otoscope. Mice were placed into a soundshielding booth, which prevented outside noise from disturbing the hearing measurement. The hearing threshold was defined as the lowest intensity at which a clear waveform was visible in the evoked trace and was determined through visual inspection of the responses.

\section{Morphological evaluation of hair cells using SEM}

SEM was used for morphological evaluation of auditory hair cells, as described previously ${ }^{32}$. Three rows of outer hair cells in a given microscopic field were evaluated. Cells were counted in three different microscopic fields of the apical, middle, and basal turns and analyzed statistically ${ }^{33}$.

\section{Transmission electron microscopy (TEM)}

Cochleae were dissected and immediately fixed with $2.5 \%$ glutaraldehyde in $0.1 \mathrm{M}$ phosphate-buffered saline (PBS), $\mathrm{pH} 7.4$, and maintained at $4{ }^{\circ} \mathrm{C}$ for $24 \mathrm{~h}$. Cochlear processing was performed for the TEM study. Cochlear specimens were post-fixed in $1 \%$ osmium tetroxide, dehydrated in graded alcohols, and embedded in Spurr's epoxy resin. Following uranyl acetate and lead citrate staining, specimens were viewed using TEM (EM 902 A; Carl Zeiss AG, Oberkochen, Germany).

\section{Hematoxylin and eosin staining}

Cochleae were fixed with $4 \%$ paraformaldehyde in PBS at $4{ }^{\circ} \mathrm{C}$ overnight. After decalcification with Calci-Clear Rapid (National Diagnostics, Atlanta, GA, USA) for 2 days and dehydration, samples were fixed in paraffin. Five series of $5-\mu \mathrm{m}$ sections were obtained using a sliding microtome (Leica, Wetzlar, Germany) in the horizontal plane parallel to the modiolus, after staining with hematoxylin and eosin for histopathology. Fibrocytes in the lateral wall and spiral ganglion cells were examined using light microscopy.

\section{TUNEL staining}

Frozen sections were dried at $37^{\circ} \mathrm{C}$ for $1 \mathrm{~h}$. Following permeabilization with $0.2 \%$ Triton $\mathrm{X}-100$ and blocking with $1 \%$ bovine serum albumin (BSA) for $30 \mathrm{~min}$, sections were washed with $0.5 \%$ BSA in PBS. Then, the sections were incubated with TUNEL reaction mixture (Roche 
Diagnostic Gmbh, Penzberg, Germany) for $1 \mathrm{~h}$ at $37^{\circ} \mathrm{C}$. After washing in PBS, the sections were mounted using VECTASHIELD mounting medium with DAPI (Vector Laboratories, Burlingame, CA, USA) and visualized with a confocal microscope (LSM710, Carl Zeiss, Jena, Germany).

\section{Cell culture}

Immortalized organ of Corti cells derived from mice ${ }^{34}$, the University of Bristol organ of Corti (UB/OC1) cells were cultured in high-glucose DMEM (Hyclone, Cramlington, UK) in the presence of $\gamma$-interferon with $10 \%$ fetal bovine serum (GIBCO, Grand Island, NY, USA) at $33^{\circ} \mathrm{C}$ in an incubator containing $10 \% \mathrm{CO}_{2}$. To induce differentiation, the cells were cultured at $37^{\circ} \mathrm{C}$ without $\gamma$ interferon under $5 \% \mathrm{CO}_{2}$.

\section{siRNA transfection}

siRNAs were synthesized by Bioneer Inc. (Bioneer, Daejeon, Korea). The target sequence for siRNAs was as follows: 5'-GAAAUGUCAACGUGUUCAA-3' for LDHB and the nonspecific-negative control (siControl). The siRNAs were transfected into UB/OC1 cells using Thermo Scientific DharmaFECT1 transfection reagent (Thermo Fisher Scientific, Waltham, MA, USA).

\section{Cell viability assay}

Cell viability was determined using the 3-(4,5-dimethylthiazol-2-yl)-2,5-dipheny-2H-tetrazolium bromide (MTT) assay (Promega, Southampton, UK). Auditory cells were treated with various concentrations of oxidative drugs and mitochondrial toxins for $24 \mathrm{~h}$. At the end of incubation, MTT solution was added to each well. Absorbance was measured using an iMark $^{\mathrm{TM}}$ Microplate Absorbance Reader (Bio-Rad, Hercules, CA, USA) at $595 \mathrm{~nm}$. All assays were performed at least three times and viability was normalized to the control.

\section{Measurement of intracellular ATP}

ATP was monitored through detection of light caused by the reaction of ATP with added luciferase and Dluciferin. Following the manufacturer's instructions for the ATPlite ${ }^{\mathrm{TM}}$ Luminescence Assay System 1000 Assay Kit (PerkinElmer, Waltham, MA, USA), cells were lysed in ATPLite lysis buffer (ATPlite: $\mathrm{NaOH}, 100$ Mriton X-100 $4 \mathrm{ml} / \mathrm{l}$ ) with shaking and sonication. The cell lysate was centrifuged at $13,000 \mathrm{rpm}$ for $5 \mathrm{~min}$ at $4{ }^{\circ} \mathrm{C}$, and the supernatant was measured. In a 96-well microplate, $65 \mu \mathrm{l}$ of dilution buffer (PBS:lysis buffer $=2: 0$ ) was mixed with $10 \mu \mathrm{l}$ of sample and $15 \mu \mathrm{l}$ of substrate solution, kept in dark conditions for $10 \mathrm{~min}$, and the luminescence intensity was measured. The ATP level was normalized to the protein concentration.

\section{Measurement of MMP}

The MMP of intact cells was determined through flow cytometry with tetramethylrhodamine methyl ester (TMRM; Molecular Probes, Life Technologies), which is a potentiometric, cell permeable fluorescent indicator that accumulates in the highly negatively charged interior of mitochondria. The culture medium was removed from adherent UB/OC1 cells, rinsed with $\mathrm{PBS}$, and resuspended in $500 \mu \mathrm{l}$ DMEM with $5 \mu \mathrm{l}$ of $1 \mathrm{mM}$ TMRM $\left(10 \mu \mathrm{M}\right.$ final concentration), and incubated at $37^{\circ} \mathrm{C}$ for $30 \mathrm{~min}$. The cells were treated with CCCP for $10 \mathrm{~min}$ prior to TMRM staining. Fluorescence was quantified using flow cytometry (FACS Vantage, Becton Dickinson).

\section{Measurement of ROS}

Intracellular ROS level was monitored through flow cytometry with the fluorescent probe $2^{\prime}, 7^{\prime}$-dichlorodihydrofluorescein (CM- $\mathrm{H}_{2} \mathrm{DCFDA}$; Molecular Probes). Cells were rinsed with $\mathrm{PBS}$ prior to pelleting and re-suspended in $500 \mu \mathrm{l} \mathrm{PBS}$ with $5 \mu \mathrm{M} \mathrm{CM}-\mathrm{H}_{2} \mathrm{DCFDA}$, then incubated at $37^{\circ} \mathrm{C}$ for $30 \mathrm{~min}$. Fluorescence was quantified using flow cytometry (FACS Vantage, Becton Dickinson).

\section{$\mathrm{NAD}^{+} / \mathrm{NADH}$ ratio assay}

Whole-cell $\mathrm{NAD}^{+}$and NADH were measured using the Elite Fluorimetric NAD/NADH ratio assay kit (eENZYME, LLC, Gaithersburg, MD, USA) according to the manufacturer's instructions. Briefly, cells were lysed with $\mathrm{NAD}^{+} / \mathrm{NADH}$ lysis buffer using a freeze-thaw cycle and sonication. The lysate was centrifuged at $13,000 \mathrm{rpm}$ and $4{ }^{\circ} \mathrm{C}$ for $15 \mathrm{~min}$ and the supernatant was collected as a sample. $\mathrm{NAD}^{+}, \mathrm{NADH}$, and $\mathrm{NAD}^{+} / \mathrm{NADH}$ ratio measurements were based on an enzymatic cycling reaction, measured using a fluorescent plate reader $(\mathrm{Ex} / \mathrm{Em}=540$ / 590). The NADH level was normalized to protein concentration.

\section{LDH activity}

Enzyme activity was determined by measuring the change in absorbance at $340 \mathrm{~nm}$, as previously descri$\mathrm{bed}^{23}$. The cells were lysed in lysis buffer $(100 \mathrm{mM}$ $\mathrm{K}_{2} \mathrm{HPO}_{4}, 30 \mathrm{mM} \mathrm{KF}, 1 \mathrm{mM}$ EDTA, and protease inhibitor cocktail) with homogenization. The lysate was centrifuged at $13,000 \mathrm{rpm}$ and $4^{\circ} \mathrm{C}$ for $15 \mathrm{~min}$, then the supernatant was collected as a sample. Spectrophotometric assays of pyruvate to lactate (P-L) were performed at $25^{\circ} \mathrm{C}$ and $\mathrm{pH}$ 7.4 (0.1 M potassium phosphate buffer), with a final $\mathrm{NADH}$ concentration of $0.25 \mathrm{mM}$, and $0.5 \mathrm{mM}$ sodium pyruvate. The assay of lactate to pyruvate (L-P) LDH activity was performed at $25^{\circ} \mathrm{C}$ and $\mathrm{pH} 8.8(50 \mathrm{mM}$ sodium pyrophosphate buffer), with final concentrations of $5.25 \mathrm{mM}$ NAD and $5 \mathrm{mM}$ lactate. In all assays, absorbance at $340 \mathrm{~nm}$ was measured at $1 \mathrm{~min}$ intervals for 
10 min with a FlexStation microplate reader. We used Vmax as the main parameter of LDH activity, normalized to protein concentration.

\section{Lactate production assay}

The lactate level in culture media was analyzed using a spectrophotometric assay, as described in a previous study $^{35}$. Briefly, $100 \mu \mathrm{l}$ of culture medium was mixed with $200 \mu \mathrm{l}$ perchloric acid and centrifuged at $3000 \mathrm{~g}$ for $15 \mathrm{~min}$; the supernatant was neutralized with potassium hydroxide $(\mathrm{KOH})(3 \mathrm{M})$ at a ratio of $400: 17$, then incubated on ice for $15 \mathrm{~min}$ and centrifuged at $3000 \times g$ for $15 \mathrm{~min}$. The supernatant was applied to the spectrometric lactate assay using a Thermo Max microplate reader (Molecular Devices Co., Sunnyvale, CA, USA). The lactate level was assessed using a standard lactate calibration curve prepared under the same conditions and expressed as lactate $(\mu \mathrm{g})$ released from $1 \mu \mathrm{g}$ of cell lysate.

\section{LDH isozyme pattern assay}

The LDH isozyme pattern was identified with nondenaturing Tris-glycine PAGE. Proteins were lysed in lysis buffer ( $100 \mathrm{mM} \mathrm{K}_{2} \mathrm{HPO}_{4}, 30 \mathrm{mM} \mathrm{KF}, 1 \mathrm{mM}$ EDTA, and protease inhibitor cocktail) with homogenization, the lysate was centrifuged at $13,000 \mathrm{rpm}$ and $4{ }^{\circ} \mathrm{C}$ for $15 \mathrm{~min}$, and the supernatant was used as a sample. Samples were kept on ice at all times. Loading samples were prepared by adding $40 \%$ sucrose with the same volume as a sample, colored with bromophenol blue, and $10-\mu \mathrm{g}$ samples were loaded in each well and separated using SDS-free Tris-glycine electrophoresis buffer. Then, the gel was stained with developer solution: lactate $(3.24 \mathrm{mg} / \mathrm{ml}), \quad \beta$-nicotinamide adenine dinucleotide $\left(\mathrm{NAD}^{+} ; 0.3 \mathrm{mg} / \mathrm{ml}\right)$, nitroblue tetrazolium (NBT; $0.8 \mathrm{mg} /$ $\mathrm{ml}$ ), and phenazine methosulfate (PMS; $0.167 \mathrm{mg} / \mathrm{ml}$ ) dissolved in $10 \mathrm{mM}$ Tris- $\mathrm{HCl}(\mathrm{pH} 8.5)$ buffer. The gel was incubated at $37^{\circ} \mathrm{C}$ for $30 \mathrm{~min}$ or longer, until the expected bands were seen, and then finished through reaction with $5 \%$ acetic acid.

\section{Western blot assay}

Total proteins were extracted using radioimmunoprecipitation assay buffer. Protein concentration was assessed with a Bio-Rad DC protein assay (Bio-Rad Laboratories, Hercules, CA, USA). Proteins were separated by electrophoresis on sodium dodecyl sulfate polyacrylamide gels. The same amount of protein $(\mu \mathrm{g})$ was loaded in each lane. After electrophoresis, the proteins were transferred to a polyvinylidene difluoride or nitrocellulose membrane and subsequently subjected to immunoblotting analysis using appropriate antibodies. The amount of loading was further determined using a western blotting housekeeping protein (lamin A, 1:5000 dilution). Using a horseradish peroxidase-conjugated secondary antibody, protein bands on the blots were visualized with enhanced chemiluminescent western blot detection reagent. Antibodies including anti-tubulin (Abcam, ab7291), anti-LDHB (Abcam, ab75167), anticomplex I (Abcam, ab110242), anti-complex II (Abcam, ab14714), anti-complex III (Abcam, ab14745), anticomplex IV (Abcam, ab14705), anti-complex V (Abcam, ab14748) were purchased from commercial company (Abcam, Cambridge, UK).

\section{Statistical analysis}

Data are expressed as mean \pm standard deviation. Significant differences between two independent groups were analyzed with Student's $t$-test using IBM SPSS Statistics for Windows (version 21.0, Amonk, NY, USA), and $P$-values $\leq 0.05$ were considered significant. When we performed $t$-test, if the two samples were satisfied with the assumption of equal variance, we used $t$-test for equal variances, whereas we applied $t$-test for unequal variances if the samples' values showed unequal variances.

\section{Acknowledgements}

This research was supported by the Basic Science Research Program through the National Research Foundation of Korea (NRF) funded by the Ministry of Education, Science, and Technology (NRF-2019R1A2C2002384) and (NRF2019R1A2C1087623).

\section{Author details}

${ }^{1}$ Department of Otolaryngology, Dali Bai Autonomous Prefecture People's Hospital, Dali, Yunnan 671000, China. ${ }^{2}$ Department of Otolaryngology, Ajou University School of Medicine, Suwon 16499, Republic of Korea. ${ }^{3}$ Institute for Medical Sciences, Ajou University School of Medicine, Suwon 16499, Republic of Korea. ${ }^{4}$ Department of Medical Sciences, Ajou University Graduate School of Medicine, Suwon 16499, Republic of Korea. ${ }^{5}$ Department of Biomedical Sciences, BK21 Plus Research Center for Biomedical Sciences, Ajou University Graduate School of Medicine, Suwon 16499, Republic of Korea. ${ }^{6}$ Department of Physiology, Ajou University School of Medicine, Suwon 16499, Republic of Korea

Conflict of interest

The authors declare that they have no conflict of interest.

\section{Publisher's note}

Springer Nature remains neutral with regard to jurisdictional claims in published maps and institutional affiliations.

Supplementary Information accompanies this paper at (https://doi.org/ 10.1038/s41419-020-2577-y).

Received: 13 October 2019 Accepted: 20 April 2020

Published online: 15 May 2020

References

1. Perez, P. \& Bao, J. Why do hair cells and spiral ganglion neurons in the cochlea die during aging? Aging Dis. 2, 231-241 (2011).

2. Hornsby, B. W., Johnson, E. E. \& Picou, E. Effects of degree and configuration of hearing loss on the contribution of high- and low-frequency speech information to bilateral speech understanding. Ear Hear. 32, 543-555 (2011).

3. Tian, $C$. et al. Red ginseng delays age-related hearing and vestibular dysfunction in C57BL/6 mice. Exp. Gerontol. 57, 224-232 (2014). 
4. Fischel-Ghodsian, N. et al. Temporal bone analysis of patients with presbycusis reveals high frequency of mitochondrial mutations. Hear Res. 110, 147-154 (1997).

5. Menardo, J. et al. Oxidative stress, inflammation, and autophagic stress as the key mechanisms of premature age-related hearing loss in SAMP8 mouse Cochlea. Antioxid. Redox Signal. 16, 263-274 (2012)

6. Short, K. R. et al. Decline in skeletal muscle mitochondrial function with aging in humans. Proc. Natl Acad. Sci. USA 102, 5618-5623 (2005).

7. Cenini, G., Lloret, A. \& Cascella, R. Oxidative Stress in Neurodegenerative diseases: from a mitochondrial point of view. Oxid. Med. Cell. Longev. 9, 2105607 (2019).

8. Luo, X., Li, R. \& Yan, L. J. Roles of pyruvate, NADH, and mitochondrial complex I in redox balance and imbalance in beta cell function and dysfunction. J. Diabetes Res. 2015, 512618 (2015).

9. Echigoya, Y., Sato, T., Itou, T., Endo, H. \& Sakai, T. Molecular characterization and expression pattern of the equine lactate dehydrogenase $\mathrm{A}$ and $\mathrm{B}$ genes. Gene 447, 40-50 (2009).

10. Zhao, D. et al. Lysine-5 acetylation negatively regulates lactate dehydrogenase A and is decreased in pancreatic cancer. Cancer Cell 23, 464-476 (2013).

11. Ren, $T$. [Determination of lactate dehydrogenase isozymes in the cochleae of guinea pigs]. Zhonghua Er Bi Yan Hou Ke Za Zhi 24(212-214), 254 (1989).

12. Kim, J. H. et al. Decreased lactate dehydrogenase B expression enhances claudin 1-mediated hepatoma cell invasiveness via mitochondrial defects. Exp. Cell Res. 317, 1108-1118 (2011)

13. Gomes, A. P. et al. Declining NAD(+) induces a pseudohypoxic state disrupting nuclear-mitochondrial communication during aging. Cell 155 1624-1638 (2013)

14. Kim, H. J. et al. Augmentation of cellular NAD+ by NQO1 enzymatic action improves age-related hearing impairment. Aging Cell 5, e13016 (2019).

15. Ciorba, A. et al. High frequency hearing loss in the elderly: effect of age and noise exposure in an Italian group. J. Laryngol. Otol. 125, 776-780 (2011).

16. Chen, Y. J. et al. Lactate metabolism is associated with mammalian mitochondria. Nat. Chem. Biol. 12, 937-943 (2016).

17. Jamesdaniel, S. et al. Targeting nitrative stress for attenuating cisplatin-induced downregulation of cochlear LIM domain only 4 and ototoxicity. Redox Biol. 10 257-265 (2016).

18. Yamasoba, T. et al. Role of mitochondrial dysfunction and mitochondrial DNA mutations in age-related hearing loss. Hear Res. 226, 185-193 (2007).

19. Rybak, L. P., Whitworth, C. A., Mukherjea, D. \& Ramkumar, V. Mechanisms of cisplatin-induced ototoxicity and prevention. Hear. Res. 226, 157-167 (2007).
20. Garrido, N. et al. Cisplatin-mediated impairment of mitochondrial DNA metabolism inversely correlates with glutathione levels. Biochem. J. 414, 93-102 (2008).

21. Pickles, J. O. Mutation in mitochondrial DNA as a cause of presbyacusis. Audio. Neurootol. 9, 23-33 (2004).

22. Bottger, E. C. \& Schacht, J. The mitochondrion: a perpetrator of acquired hearing loss. Hear. Res. 303, 12-19 (2013).

23. Someya, S. et al. Sirt3 mediates reduction of oxidative damage and prevention of age-related hearing loss under caloric restriction. Cell 143, 802-812 (2010).

24. Krieg, A. F., Rosenblum, L. J. \& Henry, J. B. Lactate dehydrogenase isoenzymes a comparison of pyruvate-to-lactate and lactate-to-pyruvate assays. Clin. Chem. 13, 196-203 (1967)

25. Datta, S. \& Chakrabarti, N. Age related rise in lactate and its correlation with lactate dehydrogenase (LDH) status in post-mitochondrial fractions isolated from different regions of brain in mice. Neurochem. Int. 118, 23-33 (2018).

26. Ross, J. M. et al. High brain lactate is a hallmark of aging and caused by a shift in the lactate dehydrogenase AVB ratio. Proc. Natl Acad. Sci. USA 107 20087-20092 (2010)

27. Washington, T. A. et al. Lactate dehydrogenase regulation in aged skeletal muscle: Regulation by anabolic steroids and functional overload. Exp. Gerontol. 57, 66-74 (2014).

28. Zhao, Y. et al. Targeting cellular metabolism to improve cancer therapeutics. Cell Death Dis. 4, e532 (2013).

29. Braidy, N. et al. Age related changes in NAD+ metabolism oxidative stress and Sirt1 activity in wistar rats. PLOS ONE 6, e19194 (2011).

30. Jiang, H. Y. et al. The dual role of poly(ADP-ribose) polymerase-1 in modulating parthanatos and autophagy under oxidative stress in rat cochlear marginal cells of the stria vascularis. Redox Biol. 14, 361-370 (2018).

31. Xiong, $\mathrm{H}$. et al. SIRT1 expression in the cochlea and auditory cortex of a mouse model of age-related hearing loss. Exp. Gerontol. 51, 8-14 (2014).

32. Choung, Y. H. et al. Korean red ginseng prevents gentamicin-induced hearing loss in rats. Laryngoscope 121, 1294-1302 (2011).

33. Viberg, A. \& Canlon, B. The guide to plotting a cochleogram. Hear. Res. 197 1-10 (2004)

34. Rivolta, M. N. \& Holley, M. C. GATA3 is downregulated during hair cell differentiation in the mouse cochlea. J. Neurocytol. 27, 637-647 (1998).

35. Lundholm, L., Mohme-Lundholm, E. \& Vamos, N. Lactic acid assay with L(plus) lactic acid dehydrogenase from rabbit muscle. Acta Physiol. Scand. $\mathbf{5 8}$ 243-249 (1963). 